Techniques, Theory and Physical Applications of the Scanning Electron Microscope and, together with Vol. II (which covers biological applications), it contains the proceedings of the SEM 1978 meetings held in Los Angeles in April 1978. These meetings were organised by SEM Inc. under the direction of Dr Om Johari, and continue the tradition of the annual symposia previously sponsored by the I.I.T. Research Institute, the proceedings of which are well known and respected by scanning electron microscopists. The present volume retains the format and maintains the high standards of the earlier publications. The papers, bibliographies and reviewers' comments contain a wealth of information for the novice and the experienced SEM user alike.

The 99 contributions include 8 review papers and 11 tutorials and cover a very wide range of topics, including specimenpreparation techniques, $\mathrm{X}$-ray microanalysis (both in the SEM and the STEM) various methods of examining semiconductor devices, energy-loss spectroscopy, Auger electron spectroscopy, backscattered electron detectors, Kossel diffraction patterns, and a bibliography on cathodoluminescence. A majority of SEM users will probably not use more than a few of the techniques described, but it is nevertheless important that one should have at least some awareness of the possibilities and limitations of the apparatus avaialable, if only so that one can realistically assess some of the more optimistic claims contained in some manufacturers' brochures!

From this reviewer's viewpoint, over $40 \%$ of the contributions were of sufficient interest to be read in some detail. The papers on X-ray microanalysis are of particular relevance, and the current widespread interest in environmental dusts is reflected in the emphasis on the identification and quantitative analysis of individual micro-particles and fibres, with papers on mounting techniques, standard materials and analysis methods with varying degrees of sophistication and accuracy. From among the other contributions, one which seems immediately worthy of further investigation is the description by Moll et al. (pp. 303-310) of a very simple (and cheap) modification which enables the secondary electron detector to be used to obtain nondirectional, back-scattered electron images at low beam current $\left(10^{\prime \prime} \mathrm{A}\right.$ range) and small spot sizes.

One small criticism is that the contributions appear to be arranged in random order. Although a rigid subdivision under subject headings would probably be unnecessarily restrictive, it would be helpful to the reader if the several papers on a single topic such as, for example, energy-loss spectroscopy or the examination of coal samples could be grouped together instead of separated by several hundred pages.

\section{S. A. BUTLER}

British Steel Corporation

Teesside Laboratories

Ladgate Lane

PO Box 74

Middlesbrough

Cleveland TS8 9EG

England

\section{J. Appl. Cryst. (1979). 12, 641}

\section{Surface crystallography by} LEED. Theory, computation and structural results. By $M$. A. Van Hove and S. Y. Tong. Pp. ix +286 . Berlin, Heidelberg, New York: Springer-Verlag, 1979. Price, DM 59.00, US \$ ca 32.50.

The chapters are (1) Introduction; (2) The physics of LEED; (3) Basic aspects of the programs; (4) Symmetry and its use; (5) Calculation of diffraction matrices for single Bravais-lattice layers; (6) The combined space method for composite layers: by matrix inversion; (7) The combined space method for composite layers: by reverse scattering perturbation; (8) Stacking layers by layer doubling; (9) Stacking layers by renormalized forward scattering (RFS) perturbation; (10) Assembling programs: the main program and the input; (11) Subroutine listings; (12) Structural results of LEED crystallography. The appendices are $(A)$ Symmetry among plane waves; (B) Lattice sums over sublattices; (C) A lineprinter plotting program.

The main part of this book explains the use of a set of computer programs that have been specially designed for surfacestructure determinations by LEED. The structures of the programs are conceived in a building-block form to allow the selection of the most efficient combination of several theoretical methods in a given situation. They include a number of features which reduce computing time and computer-store requirements. They provide a practical technique for using available symmetries of bulk and overlayer (or underlayer) lattices. The authors emphasize that the programs include the use of the combined space method which removes many limitations on the kind of surface the calculation can handle. As many as fifty subroutines in Fortran are presented in Chapter 11. Detailed explanations of all variables and arrays appearing in each subroutine are given by many lines of comment which are helpful in explaining the internal organization of the programs. In order to give the user of the programs some guidelines as to which of the various possible methods to use, and what kind of values to give for input parameters in a given situation, several sample programs are presented in Chapter 8. They will be especially useful as starting points to generate programs to be used for other surface structures. It is also convenient for readers that Chapter 12 is devoted to describing and referencing many surface structures so far confirmed by LEED investigations. The authors supplement the basic theory with a more phenomenological description of the diffraction of electrons at surfaces.

The detailed derivations of the formulae which are used for calculations are not given in this book. The readers may have to consult some of the literature cited. It will be a little inconvenient for readers that indexing of basic variables and arrays is not provided. Indexes of subroutines are provided in addition to their listings.

The programming for surface structure analysis by LEED has been considered a hard and laborious task for experimentalists. Such a book as this has long been needed. It is hoped that this book will encourage the LEED experimentalists to prepare their own programs, and to extend further applications of LEED and other related fields.

YASUYOSHI WATANABE

Departments of Physics

Faculty of Science

Chiba University

1-33 Yayoicho

Chiba

260 Japan

\section{J. Appl. Cryst. (1979). 12, 641-642}

High pressure science and technology: 6th AIRAPT conference. Vols I \& II. Edited by $K$. $D$. Timmerhaus and $M$. $S$. Barker. Pp. Vol I, xxvi +1053 ; Vol. II, $x x i i+1025$. New York: Plenum Press, 1979. Price US $\$ 75.00$ each (separately), \$ 140.000 (Both volumes together).

This book collects the papers that were 